\title{
Estudo cefalométrico comparativo dos espaços naso e bucofaríngeo nas más oclusões Classe I e Classe II, Divisão 1, sem tratamento ortodôntico, com diferentes padrões de crescimento*
}

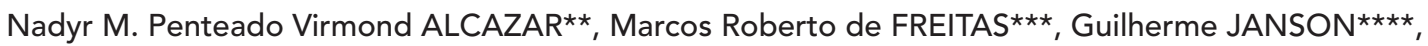

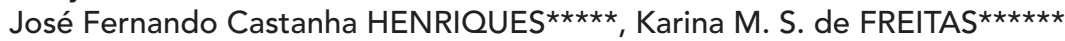

\section{Resumo}

A finalidade deste estudo foi comparar os espaços aéreos naso e bucofaríngeo em indivíduos com má oclusão Classe I e Classe II, divisão 1, segundo Angle, do gênero masculino e feminino, com idade média de 11 anos e 6 meses, com padrão de crescimento normal e vertical, não tratados ortodonticamente. A amostra desse estudo foi dividida em dois grupos: 40 pacientes apresentando Classe I e 40 pacientes com Classe II, divisão 1, cada grupo subdividido de acordo com o padrão de crescimento facial: normal e vertical. Os espaços aéreos naso e bucofaríngeo foram avaliados segundo a análise de McNamara Jr., pelas medidas NFa-NFp e BFa-BFp. A análise dos resultados obtidos revelou que, a medida do espaço bucofaringeo para Classe I com padrão de crescimento vertical e para o espaço nasofaríngeo para Classe II com padrão normal de crescimento apresentaram-se semelhantes à medida padrão da amostra de McNamara Jr.. As outras medidas apresentaram-se estatisticamente menores. Na comparação entre os grupos, o espaço nasofaríngeo no grupo Classe I com padrão de crescimento vertical, apresentou-se menor do que nos grupos Classe I e grupo Classe II divisão 1, ambos com padrão de crescimento normal. O espaço bucofaríngeo não sofreu alteração significante de um grupo para outro. Em relação à hipertrofia da tonsila faringeana, apenas o grupo Classe I com padrão de crescimento vertical apresentou obstrução; para hipertrofia das tonsilas palatinas, apenas o grupo Classe I com padrão de crescimento vertical e Classe II com padrão de crescimento normal apresentou hipertrofia das tonsilas palatinas.

Palavras-chave: Má oclusão. Cefalometria. Análise de McNamara.

* Resumo da dissertação apresentada à Faculdade de Odontologia de Bauru-USP, para obtenção do título de Mestre em Ortodontia

** Especialista e Mestre em Ortodontia, pela Faculdade de Odontologia de Bauru, da Universidade de São Paulo.

*** Professor Associado e Chefe do Departamento de Odontopediatria e Saúde Coletiva da Faculdade de Odontologia de Bauru, da Universidade de São Paulo; orientador deste trabalho.

**** Professor Associado do Departamento de Odontopediatria e Saúde Coletiva da Faculdade de Odontologia de Bauru, da Universidade de São Paulo.

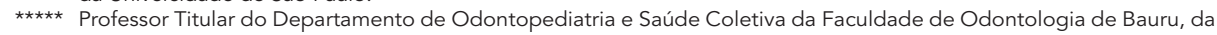
Universidade de São Paulo.

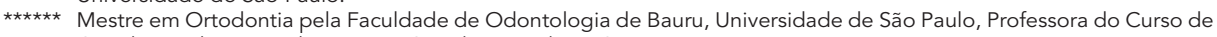
Ortodontia de Especialização em Ortodontia pela APCD-Bauru. 


\section{INTRODUÇÃO}

A relação entre a hereditariedade e o meio ambiente produz o nível de desenvolvimento biológico que um indivíduo é capaz de alcançar $^{37,5}$. Deve-se ter bem claro que a função tem a capacidade, ou não, de determinar em sua melhor forma o potencial que a hereditariedade determi-

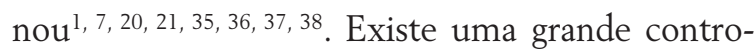
vérsia quanto à influência funcional e hereditária na dentadura como fator etiológico da má oclusão, especialmente sabendo-se que eles interagem ${ }^{8}$.

Entre os fatores funcionais que podem alterar o desenvolvimento e crescimento facial normal está a respiração predominantemente bucal. A boca não foi feita para respirar, por não estar anatômica e fisiologicamente adaptada para isto ${ }^{41}$.

A obstrução respiratória crônica, que leva à respiração bucal e causa alterações morfológicas dentofaciais, pode ser produzida por inflamação prolongada da mucosa nasal associada com alergias ou infecções crônicas, estreitamento da passagem nasal anterior, hipertrofia das tonsilas palatinas e tonsila faringeana, aumento dos cornetos, desvio de septo nasal, atresia das coanas, hipertrofia das conchas nasais e tumores no nariz ou nasofaríngeo $^{6,17,18,31,32,44}$. Além disso, a respiração bucal pode ser um hábito, isto é, ocorre sem a presença de obstrução nasal ${ }^{23,35}$.

A respiração bucal pode levar a uma série de influências prejudiciais ao processo dinâmico de crescimento e desenvolvimento dentofacial. Essas influências são alterações compensatórias ao ajuste neuromuscular para o novo padrão respiratório ${ }^{12,14,16}$.

A hipertrofia da tonsila faringeana e das tonsilas palatinas, podem obstruir os espaços naso e bucofaríngeo e ser uma das causas da respiração bucal ${ }^{28}$.

A hipertrofia das tonsilas palatinas raramente obstruem o espaço bucofaríngeo; quando isso ocorre a língua se posiciona anteriormente, mantendo suficientemente aberto o espaço respiratório faríngeo. Esse posicionamento anterior da língua pode levar a certas condições clínicas, tais como prognatismo mandibular, mordida cruzada anterior, ou protrusão alveolar dos dentes ${ }^{6,24,36,41}$.

O tamanho absoluto da tonsila faringeana não é tão importante para indicar a obstrução aérea quanto o espaço disponível que ela deixa livre na nasofarínge, que está relacionado com a morfologia esquelética da faringe.

O importante na avaliação dessa obstrução é analisar o espaço nasofaríngeo livre, estabelecendo a relação entre o tecido adenoideano e a parede faringeana circundante, onde o ar irá passar ${ }^{27,6,9}$. Alguns autores utilizam a telerradiografia cefalométrica em norma lateral para determinar esse espaço, pela medida linear da parede posterior da faringe até a parede mais anterior do palato mole ${ }^{11,24,34,42}$ outros usam fórmulas para quantificar a dimensão do espaço aéreo nasal ${ }^{15,19}$ e ainda se pode avaliar visualmente na telerradiografia ${ }^{4}$.

Sendo assim, vemos a importância de comparar as diferenças que possam existir nas vias aéreas superior e inferior de pacientes Classe I e Classe II com padrões de crescimento normal e vertical e detectar se existe comprometimento dos espaços naso e bucofaríngeo e se isso causou alterações nos arcos dentários e no crescimento e desenvolvimento craniofacial como citado na literatura ${ }^{26}$.

\section{REVISÃO DE LITERATURA}

Desde 1949, o rinologista Neivert ${ }^{31}$ relacionou as possíveis causas da dificuldade respiratória que ocasionariam alterações crônicas e também a respiração bucal.

A partir de então vários trabalhos foram publicados sobre a obstrução dos espaços naso e bucofaríngeo e os fatores advindos desta obstrução.

O fator hereditariedade é o fator primário para o desenvolvimento craniofacial mas fatores ambientais podem alterar este desenvolvimento, entre eles problemas respiratórios, como citam Enlow $^{8}$, Ferreira ${ }^{10}$, Graber ${ }^{12}$, Moss $^{29}$, Proffit ${ }^{35}$, Robert Ricketts ${ }^{38}$.

Athanasios e Athanasiou ${ }^{3}$, descrevem a faringe como um tubo fibromuscular que se estende da base do crânio até o nível da sexta vértebra cervical, onde 
continua com o esôfago. Divide-se em nasofaringe, porção superior onde se encontra a tonsila faringeana; orofaringe, porção média da faringe onde se encontram as tonsilas palatinas; laringofaringe, porção inferior da faringe. Devido à sua importância na função respiratória, a nasofaringe e a bucofaringe representam o segmento da faringe relacionada à Ortodontia.

As obstruções nasais ou nasofaringeanas, podem ser temporárias, como um resfriado comum, ou permanentes segundo Van der Linder ${ }^{49}$. Estas obstruções levariam a mudanças posturais que segundo Ackerman e Klapper ${ }^{1}$, Harvold et al. ${ }^{14}$, McNamara $\mathrm{Jr}^{25}{ }^{25}$, Mocelin ${ }^{28}$, Robert Ricketts ${ }^{38,} 39$, Subtelny ${ }^{47}$, seriam: cabeça flexionada para trás, rotação da mandíbula no sentido horário, lábios entre abertos, hipotonia do lábio superior, língua em posição anterior e inferior. Estas alterações provocam atresia do arco superior, mordida cruzada posterior uni ou bilateral, mordida aberta anterior, incisivos vestibularizados, palato profundo, aumento da altura facial anterior inferior, aumento do ângulo goníaco, sobressaliência, retrognatismo mandibular. A hipertrofia das tonsilas palatinas, como citam Athanasios e Athanasiou ${ }^{3}$, McNamara $\mathrm{Jr}^{24}$, ocupa espaço que seria ocupada pela parte posterior da língua, o que pode levar a uma posição mais anterior da língua, que esta normalmente relacionada a uma mordida cruzada anterior dento alveolar, protrusão alveolar dos dentes, mordida aberta anterior.

Alguns autores relacionam a respiração bucal com a má oclusão Classe II divisão 1, entre eles Angle ${ }^{2}$, Paul e Nanda ${ }^{33}$, Subtelny ${ }^{46}$.

Segundo Coccaro e Coccaro ${ }^{5}$, Diamon ${ }^{6}$, Handelman e Osborne ${ }^{13}$, Oulis et al. ${ }^{32}$, Silva Filho ${ }^{42}$, Subtelny ${ }^{45}$, a adenóide cresce rapidamente dos 3 aos 4 anos, ocupando metade da cavidade nasofaríngea e alcança o pico pelos 10/11 anos, ou mais tardar, aos 14/15 anos. A partir da adolescência o tecido adenoideano inicia um processo de atrofia, e a partir dessa fase o espaço nasofaríngeo tornase mais amplo. Qualquer desequilíbrio entre esse aumento nas dimensões das vias aéreas e o crescimento da adenóide poderá resultar numa permea- bilidade reduzida e obstrução nasofaríngea.

Os fatores obstrutivos podem ser múltiplos tais como rinite alérgica, edema da mucosa nasal causada por processos alérgicos, desvio de septo, atresia das coanas, hipertrofia da tonsila faringeana e das tonsilas palatinas como citam Jorge ${ }^{17}$, Oulis et al. ${ }^{32}$, Ricketts ${ }^{39}$, Trask, Shapiro, Shapiro ${ }^{48}$, observaram ainda que os fatores obstrutivos podem estar localizados na bucofaringe, nas cavidades nasais e na nasofaringe.

Os autores Ackerman e Klapper ${ }^{1}$, Cabrera A., e Cabrera M. ${ }^{4}$, Linder-Aronson e Backstrom ${ }^{23}$, Silva Filho et $\mathrm{al}^{42}$, observaram que a anatomia individual influência na alteração do espaço aéreo. O espaço aéreo nasofaríngeo compreende a distância entre o contorno anterior da tonsila faringeana e o contorno superior do palato mole. Nos pacientes braquifaciais esse espaço tende a ser maior e vai reduzindo em direção ao padrão dolicofacial. Naturalmente, a estrutura óssea nasofaríngea acompanha a morfologia facial.

Segundo Handelman e Osborne ${ }^{13}$, LinderAronson e Leighton ${ }^{22}$, Silva Filho et al. ${ }^{42}$, o crescimento da nasofaringe reflete padrões diferentes para masculino e feminino. Parâmetros para crescimento representando o tecido esquelético e a via aérea foram maiores no gênero masculino, mas menores para tecido mole.

Fujioka et al. ${ }^{11}$, Poole, Engel e Chaconas ${ }^{34}$, McNamara $\mathrm{Jr}^{24}$, relatam a validade da razão da adenóide-nasofarínge feita pela medida linear do espaço nasofaríngeo e da adenóide pela radiografia lateral da cabeça, obtendo-se assim o tamanho e potencial do espaço nasofaríngeo.

\section{PROPOSIÇÃO}

A proposta deste estudo foi avaliar se ocorreu diferença nos espaços naso e bucofaríngeo em pacientes com má oclusão Classe I e a Classe II, divisão 1, com padrão de crescimento normal e vertical; se os grupos estudados apresentaram diferenças estatisticamente significantes comparadas com os valores obtidos por McNamara Jr. ${ }^{24}$, 
que são usados como medida padrão; e segundo os valores de McNamara $\mathrm{Jr}^{24}$, se ocorreu presença de obstrução das vias aéreas superiores e inferiores nestes grupos.

\section{MATERIAL E MÉTODO Material}

A amostra deste estudo consiste de 80 telerradiografias em norma lateral de 80 pacientes, de ambos os gêneros, pertencentes à faixa etária entre 8 e 15 anos sendo a idade média 11 anos e 6 meses, sem histórico de cirurgia de tonsilas palatinas e/ou tonsila faringeana, sem tratamento ortodôntico, respiradores nasais e respiradores bucais. A amostra foi dividida em quatro grupos de 20 adolescentes cada. No grupo 1 foram incluídos os pacientes Classe I com padrão normal de crescimento. No grupo 2 foram incluídos os de Classe I com padrão vertical de crescimento. No grupo 3 foram incluídos pacientes Classe II, divisão 1, com padrão normal de crescimento, e no grupo 4 foram incluídos os de Classe II, divisão 1, com padrão vertical de crescimento.

As Classes I e II foram constatadas por meio das fichas clínicas e de modelos de estudo e o padrão de crescimento foi determinado pelas medidas FMA, S-N.Go-Gn e S-N.Gn.

\section{Método}

As radiografias selecionadas foram obtidas em norma lateral. Por meio de negatoscópio, traçouse o cefalograma padrão. Primeiramente, foram traçadas as estruturas anatômicas e em seguida foram demarcados os pontos cefalométricos de interesse (Fig. 1), que foram os pontos cefalométricos propostos por McNamara $\mathrm{Jr}^{24}$ para a análise do espaço aéreo superior e inferior, que irão determinar as distância lineares dos espaços naso e bucofaríngeo. (Tab. 1)

1) Distância linear NFa-NFp (espaço nasofaríngeo)

Também chamada via aérea superior. A largura é medida linearmente do ponto mais próximo da metade anterior do palato mole à parede faríngea posterior. É nesse local onde se localiza a tonsila faringeana (adenóides), diminuindo a largura da nasofaringe.

Valores normais segundo McNamara Jr. ${ }^{24}$ :

9 anos: $13 \mathrm{~mm}$

11 anos: $14 \mathrm{~mm}$

adulto: $17,4 \mathrm{~mm}$

2) Distância linear BFa-BFp (espaço bucofaríngeo)

Também chamada via aérea inferior. Avaliada pela largura da faringe no ponto onde, radiograficamente, a borda posterior da língua, cruza com a borda inferior da mandíbula até o ponto mais próximo da parede posterior da faringe. É neste local que se localiza as tonsilas palatinas (amígdalas).

Valores normais segundo McNamara $\mathrm{Jr}^{24}$ :

9 anos: $11 \mathrm{~mm}$

11 anos: $12 \mathrm{~mm}$

adulto: 12 a $13 \mathrm{~mm}$

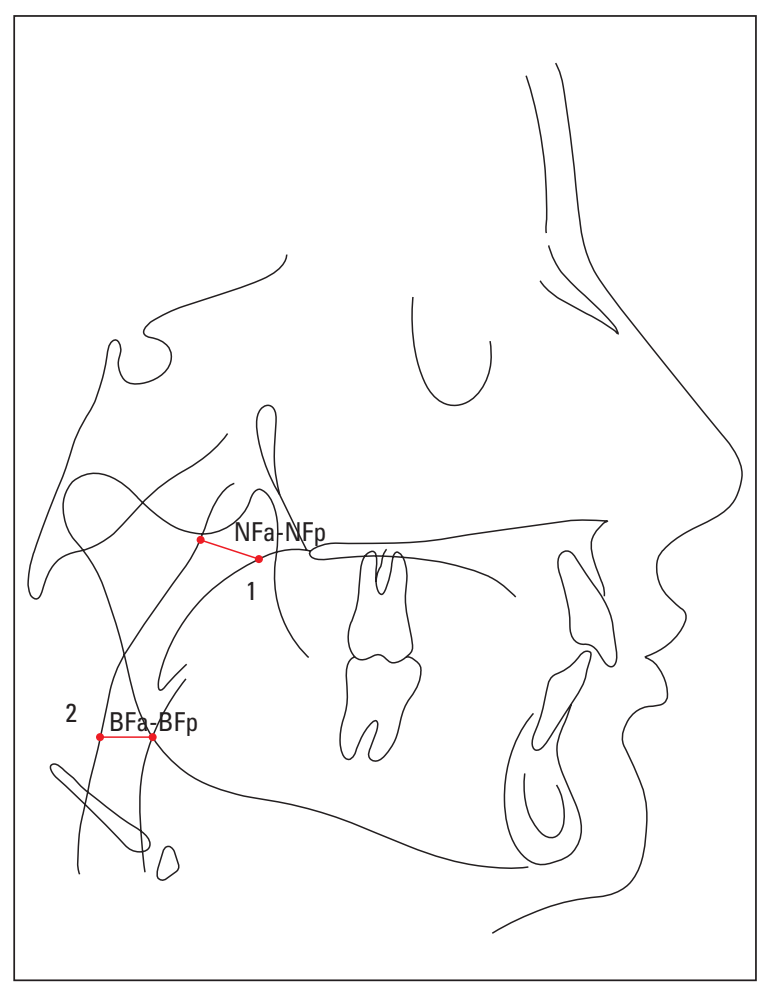

FIGURA 1 - 1) Espaço nasofaríngeo: distância linear entre NFa (nasofaríngeo anterior) e NFp (nasofaríngeo posterior). 2) Espaço bucofaríngeo: distância linear entre BFa ( bucofaríngeo anterior) e BFp( bucofaríngeo posterior). 
Tabela 1 - Valores das medidas lineares em milímetros dos espaços naso e bucofaríngeo dos quatro grupos (idade em meses)

\section{CLASSE I NORMAL}

\begin{tabular}{|c|c|c|c|}
\hline GRUP0 1 & Idade & NFa-NFp & BFa-BFp \\
\hline S1 & 152 & 9,73 & 9,73 \\
\hline S2 & 172 & 12,38 & 7,96 \\
\hline S3 & 144 & 13,05 & 8,55 \\
\hline S5 & 116 & 12,38 & 7,96 \\
\hline S6 & 158 & 12,15 & 5,40 \\
\hline S7 & 132 & 11,50 & 9,29 \\
\hline S9 & 153 & 12,38 & 11,50 \\
\hline S10 & 145 & 14,4 & 11,7 \\
\hline S12 & 152 & 12,38 & 11,50 \\
\hline S13 & 141 & 12,60 & 9,00 \\
\hline S14 & 174 & 10,80 & 9,00 \\
\hline S15 & 137 & 16,84 & 11,06 \\
\hline S16 & 141 & 11,70 & 7,65 \\
\hline S17 & 158 & 12,38 & 10,61 \\
\hline S18 & 159 & 14,60 & 8,84 \\
\hline S19 & 153 & 14,85 & 10,80 \\
\hline & 172 & 12,60 & 7,20 \\
\hline & 123 & 12,83 & 11,50 \\
\hline & 126 & 15,04 & 10,61 \\
\hline & & 9,00 & \\
\hline & 157 & & \\
\hline
\end{tabular}

\section{CLASSE II NORMAL}

\begin{tabular}{|c|c|c|c|}
\hline GRUPO 3 & Idade & NFa-NFp & BFa-BFp \\
\hline S1 & 98 & 7,96 & 15,92 \\
\hline S2 & 119 & 10,17 & 4,42 \\
\hline S3 & 164 & 17,10 & 10,80 \\
\hline S4 & 110 & 15,04 & 9,73 \\
\hline S5 & 126 & 7,96 & 12,38 \\
\hline S6 & 136 & 11,06 & 11,50 \\
\hline S7 & 154 & 11,70 & 7,20 \\
\hline S8 & 138 & 11,06 & 9,73 \\
\hline S9 & 131 & 13,05 & 11,70 \\
\hline $\mathrm{S} 10$ & 105 & 6,07 & 9,73 \\
\hline S11 & 171 & 15,92 & 4,42 \\
\hline S12 & 170 & 11,50 & 6,19 \\
\hline S13 & 131 & 12,31 & 9,73 \\
\hline S14 & 173 & 13,95 & 8,10 \\
\hline S15 & 113 & 11,06 & 11,06 \\
\hline S16 & 146 & 15,04 & 8,84 \\
\hline S17 & 172 & 22,50 & 14,40 \\
\hline S18 & 150 & 14,15 & 12,38 \\
\hline S19 & 170 & 13,27 & 9,29 \\
\hline S20 & 129 & 11,50 & 12,38 \\
\hline
\end{tabular}

CLASSE I VERTICAL

\begin{tabular}{|c|c|c|c|}
\hline GRUPO & Idade & NFa-NFp & BFa-BFp \\
\hline S1 & 123 & 13,27 & 8,84 \\
\hline S2 & 131 & 10,17 & 7,96 \\
\hline S3 & 179 & 9,73 & 10,61 \\
\hline S4 & 170 & 16,81 & 14,15 \\
\hline S5 & 156 & 6,19 & 16,81 \\
\hline S7 & 126 & 7,52 & 11,50 \\
\hline S8 & 107 & 8,84 & 14,15 \\
\hline S10 & 99 & 6,19 & 5,75 \\
\hline S11 & 155 & 16,81 & 10,61 \\
\hline S12 & 134 & 7,96 & 7,07 \\
\hline S14 & 134 & 6,19 & 15,92 \\
\hline S15 & 157 & 5,75 & 10,17 \\
\hline S16 & 106 & 8,84 & 14,15 \\
\hline S17 & 131 & 8,84 & 8,84 \\
\hline S18 & 110 & 9,73 & 13,27 \\
\hline & 119 & 15,92 & 3,53 \\
\hline & 97 & 1,76 & 10,61 \\
\hline & 117 & 9,73 & 15,92 \\
\hline & 136 & 5,75 & 7,96 \\
\hline
\end{tabular}

\section{CLASSE II VERTICAL}

\begin{tabular}{|c|c|c|c|}
\hline GRUP0 4 & Idade & NFa-NFp & BFa-BFp \\
\hline S1 & 137 & 11,50 & 7,96 \\
\hline S2 & 129 & 13,05 & 9,00 \\
\hline S3 & 119 & 6,19 & 7,07 \\
\hline S4 & 161 & 11,25 & 9,90 \\
\hline S5 & 150 & 12,38 & 8,84 \\
\hline S6 & 170 & 17,10 & 5,40 \\
\hline S7 & 114 & 12,83 & 8,84 \\
\hline S8 & 111 & 9,90 & 9,00 \\
\hline$S 9$ & 156 & 11,50 & 8,84 \\
\hline S10 & 128 & 9,73 & 7,52 \\
\hline S11 & 133 & 14,40 & 12,60 \\
\hline S12 & 164 & 10,17 & 7,07 \\
\hline S13 & 179 & 10,61 & 9,73 \\
\hline S14 & 114 & 6,19 & 9,29 \\
\hline S15 & 97 & 10,17 & 5,30 \\
\hline S16 & 95 & 7,96 & 8,84 \\
\hline S17 & 119 & 8,84 & 10,61 \\
\hline S18 & 128 & 8,10 & 7,20 \\
\hline S19 & 131 & 11,06 & 9,29 \\
\hline S20 & 178 & 10,61 & 7,52 \\
\hline
\end{tabular}




\section{Erro do Método}

Para a avaliação do erro metodológico intraexaminador, selecionou-se aleatoriamente 25\% do número total de telerradiografias por grupo ( 5 telerradiografias por grupo) sendo que a autora traçou o cefalograma das mesmas duas vezes, com um intervalo de 10 dias entre um e outro.

\section{Erro Casual}

O erro casual foi obtido com a aplicação da fórmula matemática proposta por Dahlberg ${ }^{51}$, sendo que não houve erro casual significante entre os traçados.

\section{Erro Sistemático}

O erro sistemático foi obtido de acordo com o teste " $t$ " pareado, ao nível de 5\% de significância. Não se observaram diferenças estatisticamente significantes entre os dois traçados.

\section{Análise Estatística}

$\mathrm{Na}$ análise estatística utilizou-se para a comparação entre os quatro grupos, a análise de variância a um critério (ANOVA). Na observação de um resultado significante, o teste de Tukey foi utilizado com o objetivo de indicar especificamente em quais grupos estaria ocorrendo essa significância; para a comparação do espaço aéreo de cada grupo com a medida padrão de McNamara Jr., aplicouse o teste "t" de Student paramétrico; para a avaliação do espaço aéreo em cada grupo analisado, foi aplicado o teste do Qui-Quadrado.

\section{RESULTADO}

$\mathrm{Na}$ avaliação das diferenças entre os quatro grupos para os espaços naso e bucofaríngeo, mostrou que o espaço nasofaríngeo (NFa-NFp), houve diferença estatísticamente significante do grupo 2 com o grupo 1 e com o grupo 3. Sendo que o grupo 2 apresentou-se com o espaço nasofaríngeo menor que o grupo 1 e o grupo 3.

Para o espaço bucofaríngeo (BFa-BFp), não houve diferença significante entre os grupos.

$\mathrm{Na}$ avaliação da comparação do espaço aéreo,
NFa-NFp e BFa-BFp, com as medidas de McNamara Jr., o grupo 1 mostrou diferença estatisticamente significante para NFa-NFp e para BFa-BFp, que foram menores do que a medida de McNamara Jr. O grupo 2 houve diferença estatísticamente significante para $\mathrm{NFa}-\mathrm{NFp}$, menor, e para $\mathrm{BFa}-\mathrm{BFp}$ não houve diferença estatisticamente significante. $\mathrm{O}$ grupo 3 não houve diferença estatisticamente significante para NFa-NFp, e para BFa-BFp observou-se diferença estatisticamente significante, para menor. O grupo 4 houve diferença estatisticamente significante para NFa-NFp e para BFa-BFp, que foram menores do que a medida de McNamara Jr.

$\mathrm{Na}$ avaliação da medida NFa-NFp para cada grupo mostrou que não houve provável presença de obstrução do espaço nasofaríngeo, apenas o grupo 2 apresentou uma porcentagem pequena de provável obstrução do espaço nasofaríngeo. $\mathrm{Na}$ avaliação da medida BFa-BFp para cada grupo mostrou que não houve provável obstrução do espaço bucofaríngeo, apenas os grupos 2 e 3 apresentaram uma porcentagem pequena de casos de provável obstrução bucofaringeana.

\section{DISCUSSÃO}

Neste estudo procuramos estabelecer várias relações dos espaços aéreos naso e bucofaríngeo em pacientes com má oclusão Classe I e Classe II, divisão 1, de Angle, com padrões de crescimento normal e vertical, na faixa etária dos 8 aos 15 anos.

Quando comparamos os grupos entre si avaliando o espaço naso e bucofaríngeo na má oclusão de Classes I e II, divisão 1, com padrão de crescimento vertical e normal, verificamos que para o espaço nasofaríngeo a maior diferença estatisticamente significante foi encontrada entre o grupo 2 com o grupo 1 e o grupo 3. Essa diferença se mostrou menor para o grupo 2 do que para os grupos 1 e 3. Na comparação do grupo 2 com o grupo 1 e o grupo 3 , pode-se inferir que, o espaço aéreo nasofaríngeo, além de estar relacionado com o tamanho da tonsila faringeana, é também influenciado pelo padrão de crescimento do indivíduo, que influencia 
o tamanho da faringe, pois para o grupo com padrão de crescimento vertical, em que a largura da nasofaringe é mais estreita e longa acompanhando a morfologia facial, temos um espaço aéreo menor. $\mathrm{Na}$ literatura encontramos vários autores que relacionam o espaço nasofaríngeo menor em pacientes com face longa e estreita, isto é, em pacientes com padrão dolicofacial como: Ackerman e Klapper ${ }^{1}$, Linder-Aronson e Backstrom ${ }^{23}$, Proffit et al. ${ }^{35}$, Silva Filho et al. ${ }^{42}$.

Quanto a comparação entre os outros grupos para o espaço NFa-NFp, não houve diferença estatisticamente significante, o que mostra que não houve uma relação do espaço nasofaríngeo com má oclusão e padrão de crescimento, o que coincide com os achados de McNamara Jr. ${ }^{25}$, Watson, Wareen e Fisher ${ }^{50}$ mas se opõe aos achados de Angle², Merger e Jacobs ${ }^{26}$, Paul e Nanda ${ }^{33}$, que encontraram relação da má oclusão e o padrão de crescimento com o espaço nasofaríngeo.

Em relação ao espaço bucofaríngeo, representado pela medida $\mathrm{BFa}-\mathrm{BFp}$, não foi observado diferença significante entre os grupos, não mostrando relação com a idade dos pacientes e nem com o padrão de crescimento e a má oclusão. Isto confirma as achados de McNamara $\mathrm{Jr}^{24}$, que mostra que o espaço bucofaríngeo não se altera com a idade, e com os achados de Martins ${ }^{30}$, Athanasiou ${ }^{3}$, que relatam alterações no espaço bucofaríngeo relacionado com a protrusão mandibular, que não esta presente na amostra deste estudo.

$\mathrm{Na}$ comparação do espaço naso e bucofaríngeo com as medidas de McNamara $\mathrm{Jr}^{24}$ encontramos uma diferença estatisticamente significante entre a amostra do nosso trabalho e o padrão da amostra de McNamara $\mathrm{Jr}^{24}$. Isto provavelmente ocorreu por ser diferente as variáveis da amostra deste trabalho com a amostra de McNamara Jr., que era constituída de um único padrão de crescimento (equilibrado) e não apresentava má oclusão. Como vimos na literatura, a faringe, onde está localizado o espaço aéreo naso e bucofaríngeo, sofre influência no seu desenvolvimento com as variáveis; padrão de crescimento, que afeta o tamanho da profundidade esquelética da faringe, o dimorfismo sexual e a má oclusão $^{1,4,8,13,22,23,26,44,47}$.

$\mathrm{Na}$ avaliação do espaço aéreo de cada grupo, foi usada como referência as medidas de McNamara $\mathrm{Jr}^{24}$, para obstrução das vias aéreas superiores e vias aéreas inferiores por ser uma medida padrão.

Segundo McNamara $\mathrm{Jr}^{24}$, um espaço menor do que $5 \mathrm{~mm}$ para NFa-NFp é provável indicação de obstrução do espaço aéreo nasofaríngeo.

Para BFa-BFp, uma medida acima de $15 \mathrm{~mm}$ indica posicionamento anterior da língua, indicativo de hipertrofia das tonsilas palatinas.

Diante dos resultados obtidos, verificou-se que para o espaço nasofaríngeo (NFa-NFp) não há presença de obstrução pela tonsila faringeana (adenóide) isso nos leva a sugerir que, mesmo tendo na amostra respiradores bucais, não foi a presença de hipertrofia da tonsila faringeana a causa dessa respiração bucal, pois o espaço nasofaríngeo não está diminuído. Isso confirma os achados de Jorge ${ }^{17}$, Neivert ${ }^{31}$, Oulis et al. ${ }^{32}$, Ricketts $^{39}$, Rubin $^{40}$, Trask, Shapiro, Shapiro ${ }^{48}$ que relacionaram outras possíveis causas para a dificuldade respiratória, como tamanho das narinas, desvio de septo, deformidades nas conchas nasais, pólipos nasais, atresia da coana, edema da membrana da mucosa do nariz devido a alergia ou sinusite crônica.

Proffit et al. ${ }^{35}$, cita que a maioria dos indivíduos com padrão de face longa não apresentam evidência de obstrução nasal, o que sugere que um padrão facial vertical não esta ligado a obstrução pela tonsila faringeana, isto reflete os achados desse estudo onde os pacientes com padrão de crescimento vertical não apresentaram obstrução da nasofaringe.

$\mathrm{Na}$ avaliação para o espaço $\mathrm{BFa}-\mathrm{BF}$, apenas o grupo 2 e o grupo 3 mostraram pequena porcentagem de casos de hipertrofia das tonsilas palatinas.

Esses achados são contraditórios em relação à literatura, onde encontramos a hipertrofia das tonsilas palatinas relacionada a protrusão mandibular 
segundo Athanasiou ${ }^{3}$, McNamara $\mathrm{Jr}^{24}$, Martins $^{30}$. Aqui a encontramos presente na má oclusão Classe I e na Classe II, divisão 1 de Angle, apesar de ser uma porcentagem muito pequena.

\section{CONCLUSÕES}

Com base nos resultados obtidos e na metodologia utilizada, pode-se concluir que:

Apenas o espaço nasofaríngeo apresentou diferença estatisticamente significante no grupo com Classe I com padrão vertical de crescimento comparando com as más oclusões de Classe I e Classe II, divisão 1, com padrões de crescimento normal, que apresentaram valores menores.

Os espaços naso e bucofaríngeo se apresentaram significativamente diferentes dos valores de McNamara Jr., cujos valores foram menores. Apenas nas más oclusões de Classe II, divisão 1, com padrão normal de crescimento, para o espaço nasofaríngeo, e nas Classe I com padrão vertical de crescimento, para o espaço bucofaríngeo, apresentaram-se estatisticamente não significante.

Não ocorreu obstrução dos espaços naso e bucofaríngeo em nenhum dos grupos avaliados.

\title{
A Comparative cephalometric study of the naso and oropharyngeal space in malocclusions Class I and Class II Division 1, without orthodontic treatment with different growth patterns
}

\begin{abstract}
The aim of this study is to compare the naso and oropharyngeal air space in people with malocclusion class I and class II division 1, according to Angle, with mean age from 8 to 15 years old with normal and vertical growth pattern not treated orthodontically. This study was divided into two groups: 40 patients with class I, and 40 with class II, division 1 subdivided according to facial growth: normal and vertical. The naso and oropharyngeal airway space was assessed according to McNamara's analysis by using the Nfa-NFp and Nba-NBp measurements. The analysis of the results obtained revealed that, the oropharyngeal measurements for class I with pattern vertical growth and nasopharyngeal class II with pattern normal growth were similar to the reference measures in McNamara's sample. The other measurements were statistically smaller. In the comparison between groups, the oropharyngeal spaces did not show a difference between one group and another. The nasopharyngeal space in the class I group with vertical growth, showed a narrower space than in the class I group and in the class II group with pattern normal growth. In relation to adenoids hypertrophy, only class I with pattern vertical growth showed obstruction; for tonsils hypertrophy, only class I with pattern vertical growth and class II with pattern normal growth showed tonsils hypertrophy.
\end{abstract}

Key words: Malocclusions. Cephalometric. Analysis of McNamara Jr.

\section{REFERÊNCIAS}

1. ACKERMAN, R. I.; KLAPPER, L. Tongue position and open-bite: the key roles of growth and the nasopharyngeal airway. J Dent Child, Chicago, v. 48, p. 339-345, Sept./ Oct.1981.

2. ANGLE, E. H. Classification of malocclusion. In: Dental Cosmos, St. Louis, v. 41, p. 246-264,1899.
3. ATHANASIOU, A. Possibilites and limitations of variable and analyses. In:_ Orthodontic cephalometry. London: Mosby-Wolfe, 1995. p. 90-92.

4. CABRERA, A. C.; CABRERA, M. C. de. Cefalometria. In: Ortodontia clínica I. Curitiba: Interativa, 2000. cap.7, p. $205-$ 206. 
5. COCCARO, P. J.; COCARRO, P. J. Dental development and the plaryngeal lymphoid tissue. Otolariyngol Clin North Am, [S I.], v. 20, no. 2, p. 242-257, May 1987.

6. DIAMOND, O. J. Tonsils and Adenoids; Why the dilemma? Am J Orthod, St. Louis, v. 78, no. 5, p. 495-503, Nov. 1980.

7. DUNN, G. F.; GREEN, L. J.; CUNAT, J. J. Relationships between variation of mandibular morphology and variotion of nasopharyngeal airway size in monozigotic twins. Angle Orthod, Appleton, v. 43, no. 2, p.129-135, Apr. 1973.

8. ENLOW, D. H.; HANS, M. Complexo nasomaxilar. In Noções básicas sobre crescimento facial. São Paulo: Ed. Santos, 1998. cap. 5, p.1-17.

9. FAIRCHILD, R. C. A pediatrician views the tonsil and adenoid problem. Am J Orthod, St. Louis, v. 54, no. 7, p. 491-494, July 1968.

10. FERREIRA, F. V. Anatomia funcional dos músculos cuticulares e mastigadores aplicações ortodônticas. Bol Soc Paul Ortop, São Paulo, v. 4, n. 2, p. 31-35, jul./dez. 1954

11. FUJIOKA, M. et al. Radiographic evaluation of adenoidal size in children: adenoidal-nasopharyngeal ratio. Am J Roentgenol, Springfield, v. 133, p. 401-404, Sept. 1979.

12. GRABER, T. M. The "three M's": muscles, malformation and malocclusion. Am J Orthod, St. Louis, v. 49, no. 6, p. 418-450, June 1963.

13. HANDELMAN, C. S.; OSBORNE,G. Growth of the nasopharynx and adenoid development from one to eighteen years. Angle Orthod, Appleton, v. 46, no. 3, p. 243-258, July 1976.

14. HARVOLD, E. P. et al. Primate experiments on oral respiration. Am J Orthod, St. Louis, v.79, no. 4, p. 359-372, Apr. 1981.

15. HINTON, V. A.; WARREN, D. W.; HAIRFIELD, W. M.; SEATON, $D$. The relationship between nasal cross-sectinal area and nasal air volume in normal and nassally impaired adults. Am J Orthod, St. Louis, v. 92, no. 4, p. 294-298, Oct. 1987.

16. HINTON, V. A. et al. Upper airway pressures during breathing: a comparison of normal and nasally incompetent subjects with modeling studies. Am J Orthod, St. Louis, v. 89, no. 6, p. 492 498, June 1986

17. JORGE, E. P. Estudo das características morfológicas, craniofaciais de pacientes com má oclusão de Classe II, divisão $1^{\text {a }}$ de Angle, com predomínio da respiração bucal. São Paulo, 2000. 202 f. Dissertação (Mestrado)-Faculdade de Odontologia, Universidade de São Paulo, São Paulo, 2002

18. JORGE, E. P.; ABRÃO, J.; CASTRO, A. B. B. A. T. de. Avaliação dos fatores obstrutivos da via aérea superior em pacientes com má oclusão de Classe II divisão 1 de Angle, por meio da vídeoendoscopia. R Dental Press Ortop Facial, Maringá, v. 6, n. 2, p. 49-58, mar./abr. 2001

19. KEALL, C. L.; VIG, P. S. An improved technique for the simultaneous measurements of nasal and oral respiration. Am J Orthod Dentofacial Orthop, Maringá, v. 91, no. 3, p. 207-212, Mar. 1987

20. KERR, W. J. S. The nasopharynx, face height, and overbite. Angle Orthod, Appleton, v. 55, no. 1, p. 31-36, Jan. 1985

21. KÖHLER, N. R. W. Disturbios miofuncionais: considerações sobre seus fatores etiológicos e consequências sobre o processo de crescimento, desenvolvimento da face. R Dental Press Ortodon Ortop Facial, Maringá, v. 5, n. 3, p. 66-79, maio/jun. 2000.

22. LINDER-ARONSON, S.; LEIGHTON, B. C. A longitudinal study of the development of the posterior nasopharyngeal wall between 3 and 16 years of age. Eur J Orthod, London, v. 5, no. 1, p. 47-58, Feb. 1983

23. LINDER-ARONSON, S.; BACKSTROM, A. A comparison between mouth and nose breathers with respect to occlusion and facial dimensions. Odontol Revy, Lund, v. 11, no. 2, p. 343-376, 1960.

24. McNAMARA Jr., J. A. A method of cephalometric evaluation. Am J Orthod, St. Louis, v. 86, p. 449-469, Dec. 1984.

25. McNAMARA Jr., J. A. Influence of respiratory pattern on craniofacial growth. Angle Orthod, Appleton, v. 51, no. 4, p. 269-300, Oct. 1981

26. MERGEN, C. D.; JACOBS, M. R. The size of nasopharynx associated with normal occlusion and Class II malocclusion. Angle Orthod, Appleton, v. 40, no. 4, p. 342-346. Oct. 1970

27. MIYASHITA, K. Contemporary cephalometric radiography Osaka: Quintessence, 1996. cap. 2, p.154-157.

28. MOCELLIN, L. Alteração oclusal em respiradores bucais. J Bras Or todon Ortop Maxilar, Curitiba, v. 2, n. 7, p. 45-48, jan./fev. 1997
29. MOSS, M. L. Vertical growth of the human face. Am J Orthod, St. Louis, v. 50, no. 5, p. 359-376, May 1964

30. MARTINS, J. C. R. D. Influência da hipertrofia amigdaliana nas más oclusões de Classe I e Classe II. Bauru, 1988. 120 f. Tese (Doutourado)-Faculdade de Odontologia de Bauru, Universidade de São Paulo, Bauru, 1988.

31. NEIVERT, H. Rhinologic experiences to aid the orthodontist. Am J Orthod, St. Louis, v. 35, no. 3, p.167-178. Mar. 1949.

32. OULIS, C. J. et al. The effect of hypertrophic adenoids and tonsils on the development of posterior crossbite and oral habits. J Clin Pediatr Dent, Birmingham, v. 18, no. 3, p.197-201, Spring 1994.

33. PAUL, J. L.; NANDA, R. S. Effect of mouth breathing on dental oclusion. Angle Orthod, Appleton, v. 43, no. 2, p. 201-206, Apr. 1973.

34. POOLE, M. N.; ENGEL, G. A.; CHACONAS, S. J. Nasopharyngeal cephalometrics. J Oral Surg, Chicago, v. 49, no. 3 p. 266-271, Mar. 1980

35. PROFFIT, W. R. et al. The etiology of orthodontic problems. In: Contemporary Orthodontics. St. Louis: [s. n.], 1986. p. 95-120.

36. PROFFIT, W. R. Equilibrum theory revisited: factors influencing position of the teeth. Angle Orthod, Appleton, v. 48, no. 3, p.175-186, July 1978

37. RAKOSI, T.; JONAS, I.; GRABER, T. M. Color Atlas of dental medicine: orthodontic diagnosis. New York: Thieme Medical 1993. p.161-162

38. RICKETTS, R. M. Respiratory obstruction syndrome. Am J Orthod, St. Louis, v. 54, p. 495-507, July 1968.

39. RICKETTS, R. M. The interdependence of nasal and oral capsules. In: McNAMARA Jr., J. A. Naso Respiratory function and craniofacial Growth. Ann Arbor: Center for Humam Growth and Development, University of Michigan, 1979. p.165-198.

40. RUBIN, R. M. Mode of respiration and facial growth. Am J Orthod, St. Louis, v. 78, no. 5, p. 504-510, Nov. 1980.

41. SCHINESTSCK, P. A. A relação entre a maloclusão dentária, a respiração bucal e as deformidades esqueléticas. Jornal Brasileiro de Ortodontia e Ortopedia Facial, Curitiba, v. 1 , n. 4, p. 45-55, jul./ago. 1996.

42. SILVA FILHO, O. G. et al. Dimensões da nasofaringe em crianças de 7 anos de idade portadoras de oclusão normal. R Ortodon São Paulo, v. 22, n. 2, p. 20-30, maio/ago. 1989

43. SIMÕES, W. A.; BRANDÃO, M. R. A língua e o complexo hióideo como recurso no diagnóstico e tratamento das má-oclusões. Ortodontia, São Paulo, v. 26, n. 2, p. 89-98, maio/ago. 1993

44. STEELE, C. H.; RICKETTS, R. M.; FAIRCHILD, M. D. Forum on the tonsil and adenoid problem in ortodontics. Am J Orthod, St. Louis, v. 54, no.7, p. 495-507, July 1968

45. SUBTELNY, J. D. The Significante of adenoid tissue in orthodontic. Angle Orthod, Appleton, v. 24, p. 59-69, 1954.

46. SUBTELNY, D. J. Malocclusion, orthodontic corrections and orofacial muscle adaptation. Angle Orthod, Appleton, v. 40, no. 3, p.170-199. Oct. 1970

47. SUBTELNY, J. D. Oral respiration: facial maldevelopment and corrective dentofacial orthopedics. Angle Orthod, Appleton, v. 50 , no. 3, p.147-164, July 1980

48. TRASK, G. M.; SHAPIRO, G. G.; SHAPIRO, P. A. The effects of perennial allergic rhinitis on dental and skeletal development: A comparison of sibling paris. Am J Orthod Dentofacial Orthop, St. Louis, v. 92, no. 4, p. 286-293, Oct. 1987

49. LINDEN, van der. Variações funcionais. In: . Crescimento e ortopedia facial. Rio de Janeiro: Quintessense, 1990. cap. 7, p.159-162.

50. WATSON, R. M.; WARREN, D. W.; FISCHER, N. D. Nasal resistence, skeletal classification, and mouth breathing in orthodontic patients. Am J Orthod, St. Louis, v. 54, no. 5, p. 367-379, May 1968

51. ZAR, J. H. Biostatical analysis. 3rd ed. New Jersey: Prentice Hall, 1996

\section{Endereço para correspondência}

Nadyr M. Virmond Alcazar

Av. Herval 606

CEP: 87013-110

Maringá-PR

E-mail: ralcazar@brturbo.com 